\title{
Low-Complexity LSMR Equalisation of FrFT-Based Multicarrier Systems in Doubly Dispersive Channels
}

\author{
Ahmed A. A. Solyman, Stephan Weiss, and John J. Soraghan \\ Centre for Signal \& Image Processing, Department of Electronic \& Electrical Engineering \\ University of Strathclyde, Glasgow G1 1XW, Scotland, UK \\ aaasolyman@Gmail.com,\{j.soraghan,stephan\}@eee.strath.ac.uk
}

\begin{abstract}
The discrete fractional Fourier transform (FrFT) has been suggested to enhance performance over DFT-based multicarrier systems when transmitting over doubly-dispersive channels. In this paper, we propose a novel low-complexity equaliser for inter-symbol and inter-carrier interference arising in such multicarrier transmission system. Due to a lower spreading in the FrFT-domain compared to the DFTchannel matrix as compared to the DFT domain, the equaliser can approximate the fractional-domain channel matrix by a band matrix. Further, we utilise the least squares minres (LSMR) algorithm in the calculation of the equalisation, which exhibits attractive numerical properties and low complexity. Simulation results demonstrate the superior performance of the proposed LSMR equaliser over benchmark schemes.
\end{abstract}

Index Terms-Multicarrier transmission, fractional Fourier Transformation, doubly dispersive channel, low complexity equalisation.

\section{INTRODUCTION}

The popularity of multicarrier systems such as orthogonal frequency division multiplexing (OFDM) is based on the ability to cancel inter-symbol-interference (ISI) without explicit knowledge of channel state information (CSI). In the case of a stationary channel, the channel effect on the received symbols can therefore be mitigated by a single equaliser coefficient per carrier. However, OFDM does not perform well if the system is not synchronised or if the channel changes during the transmission of one OFDM symbol. In such a time-varying scenario, OFDM suffers from inter-carrier-interference (ICI), resulting in the need for equalisation [1-3].

The inclusion of an equaliser increases complexity in terms of receiver processing and requires the estimation of the channel matrix including all subcarrier gains as well as all ICI-generating terms. The main advantage of OFDM to permit simple processing with only per-carrier single-tap equalisers is therefore negated. In fact, the complexity and CSI requirements may be sufficiently high to favour optimal filter bank based methods with superior performance over OFDM [4, 5].

Recently, the standard DFT/FFT in OFDM systems has been replaced by discrete versions of the fractional Fourier transform (FrFT) [6-9]. While the FrFT, unlike the DFT in classical OFDM, does not diagonalise the circulant channel matrix found in the case of transmission over stationary channels, it concentrates the coefficients near the diagonal. In doubly-dispersive channels, the channel matrix of a classical DFT-OFDM system is no longer diagonal and coefficients can spread far, while the matrix of the FrFT system still retains most of its energy near the diagonal. As a result, performances for FrFT systems have been reported to be superior to classical OFDM in doubly-selective channels [6-9].

Various DFT-domain equalisation methods have been proposed in the context of OFDM, including zero-forcing (ZF) and minimum mean-square error (MMSE) schemes [10] as well as successive interference cancellation (SIC) [11]. In all these schemes the equaliser complexity can be reduced by exploiting the approximate band structure of the resulting channel matrix [10, 12]. ICI equalisers in [13, 14] apply the LSQR algorithm [15, 16] which offers a low complexity approach to solve linear systems.

In this paper, we focus on FrFT-OFDM systems in doublydispersive channels, and apply low-cost equalisation schemes developed for classical OFDM systems such as in [17]. We propose a band matrix approximation, which exploits the increased energy concentration of the channel matrix within the FrFT as compared to a classical OFDM system. Further, our low cost equaliser uses a very recently proposed least squares minres (LSMR) approach [18], which is an iterative algorithm that promises better numerical stability and faster convergence compared to LSQR.

The remainder of this paper is organised as follows. In Sec. II we review the FrFT and the overall FrFT-OFDM system model. In Sec. III we describe the proposed ICI equalisation method using the LSMR algorithm. Simulation results are provided in Sec. IV that compare the performance of the new structure to existing methods. Sec. V concludes the paper.

\section{FRFT-OFDM SYSTEM MODEL}

\section{A. The fractional Fourier transform and its Discretisation}

The FrFT is the generalised formula for the Fourier transform that maps a function into an intermediate domain between time and frequency, and may be interpreted as a rotation operator in the time-frequency plane. This property makes the FrFT especially suited for the processing of linear frequency modulated (LFM) or chirp-like signals. The FrFT of order $a$ of an arbitrary function $x(t)$, with an angle $\alpha=a \pi / 2, a \in \mathbb{R}$, is defined as [19]

$$
X_{\alpha}(u)=\int_{-\infty}^{\infty} x(t) K_{\alpha}(t, u) \mathrm{d} t
$$




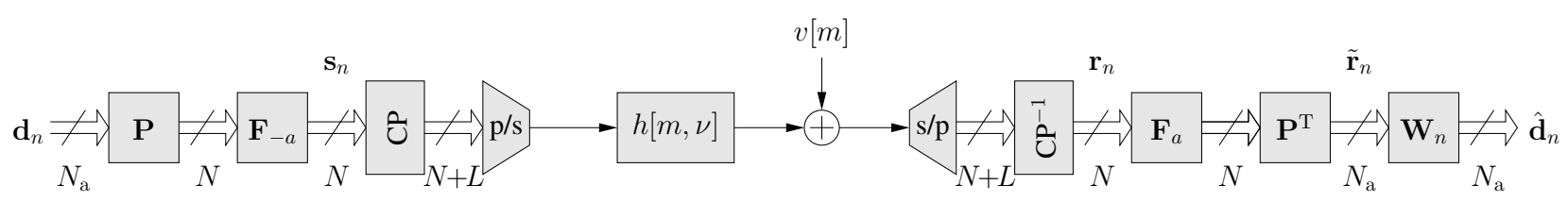

Figure 1. FrFT-based OFDM system, whereby the DFrFT replaces the DFT in a classical OFDM setup [6]. The proposed system features a low-cost equaliser matrix $\mathbf{W}_{n}$.

The transform kernel $K_{\alpha}(t, u)$ is given by

$$
K_{\alpha}(t, u)= \begin{cases}A_{\alpha} e^{\omega_{\alpha}} & \alpha \neq n \pi \\ \delta(t-u) & \alpha=n 2 \pi \\ \delta(t+u) & \alpha+\pi=n 2 \pi\end{cases}
$$

where $A_{\alpha}=\sqrt{\frac{1-j \cot \alpha}{2 \pi}}$ and $\omega_{\alpha}=j \frac{t^{2}+u^{2}}{2} \cot \alpha-j u t \csc \alpha$. The inverse FrFT (IFrFT) can be expressed as

$$
x(t)=\int_{-\infty}^{\infty} X_{\alpha}(u) K_{\alpha}(t, u) \mathrm{d} u
$$

With (2), the FrFT can be seen as a description of $x(t)$ within a basis formed by orthonormal LFM functions in the $u$ or fractional Fourier domain. The time and frequency domains can be considered as special cases when $a=0$ and $a=1$, respectively.

Different discretations of (1) and (3) have been proposed, varying in accuracy and complexity. In our work, we select the discrete FrFT (DFrFT) proposed in [19] to ensure that the transform kernel of the DFrFT and its inverse transform are orthogonal and reversible. The DFrFT formula of a data segment $x[n]$ is defined as

$$
X_{a}[k]=\sum_{n=-N / 2}^{N / 2} x[n] K_{a}[n, k],
$$

or in vector notation

$$
\mathbf{y}_{a}=\left[\begin{array}{c}
X_{a}[0] \\
X_{a}[1] \\
\vdots \\
X_{a}[N-1]
\end{array}\right]=\mathbf{F}_{a}\left[\begin{array}{c}
x[0] \\
x[1] \\
\vdots \\
x[N-1]
\end{array}\right]=\mathbf{F}_{a} \mathbf{x}
$$

The N-point DFrFT matrix $\mathbf{F}_{a}$ is unitary, therefore admitting a straightforward inverse discrete FrFT (IDFrFT) as $\mathbf{x}=$ $\mathbf{F}_{a}^{\mathrm{H}} \mathbf{y}_{a}=\mathbf{F}_{-a} \mathbf{y}_{a}$, where $(\cdot)^{\mathrm{H}}$ denotes Hermitian transpose.

\section{B. FrFT-Based OFDM System}

A conventional OFDM systems applies an inverse discrete Fourier transform (IDFT) matrix to a data vector $\mathbf{s}_{n}$ and introduces a cyclic prefix (CP) of length $L$ prior to multiplexing the OFDM symbol across a dispersive channel $h[n, \nu]$ corrupted by additive white Gaussian noise $v[n]$. After demultiplexing the received signal and removal of the cyclic prefix, a DFT matrix reconstructs the transmitted data vector $\hat{\mathbf{s}}_{n}$. In a DFrFTbased OFDM system, the DFT matrix is replaced by a DFrFT matrix $\mathbf{F}_{a}[6,20]$ as shown in Fig. 1.
The multiplexed signal $\mathbf{r}_{n}=\mathbf{H}_{n} \mathbf{s}_{n}+\mathbf{v}_{n}$ after cyclic prefix removal in Fig. 1 is characterised by a system matrix with elements

$$
\left[\mathbf{H}_{n}\right]_{i, j}= \begin{cases}h[n-L+i, i-j] & i \geq j, \\ h[n-L+i, L+i-j-1] & i<j .\end{cases}
$$

In stationary conditions, $\mathbf{H}_{n}$ is circulant, and can be decoupled by $\mathbf{F}_{a}$ with $a= \pm 1$, whereby the case $a=1$ represents the conventional OFDM system.

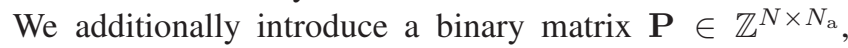
which assigns a data vector $\mathbf{d}_{n} \in \mathbb{C}^{N_{\text {a }}}$ to $N$ subcarriers, of which only $N_{\mathrm{a}}$ are active according to

$$
\mathbf{P}=\left[\begin{array}{lll}
\mathbf{0}_{N_{\mathrm{a}} \times\left(N-N_{\mathrm{a}}\right) / 2} & \mathbf{I}_{N_{\mathrm{a}}} & \mathbf{0}_{N_{\mathrm{a}} \times\left(N-N_{\mathrm{a}}\right) / 2}
\end{array}\right]^{\mathrm{T}},
$$

where $\mathbf{0}_{L \times M}$ is an $L \times M$ matrix with zero entries, and $\mathbf{I}_{L}$ an $L \times L$ identity matrix. The equaliser matrix $\mathbf{W}_{n} \in \mathbb{C}^{N_{\mathrm{a}} \times N_{\mathrm{a}}}$ in the receiver operates on the input

$$
\begin{aligned}
\tilde{\mathbf{r}}_{n} & =\mathbf{P}^{\mathrm{H}} \mathbf{F}_{a} \mathbf{H}_{n} \mathbf{F}-a \\
& =\mathbf{C}_{n, a} \mathbf{d}_{n}+\tilde{\mathbf{v}}_{n}+\mathbf{P}^{\mathrm{H}} \mathbf{F}_{a} \mathbf{v}_{n}
\end{aligned}
$$

with a system matrix $\mathbf{C}_{n, a} \in \mathbb{C}^{N_{\mathrm{a}} \times N_{\mathrm{a}}}$. The purpose of the binary matrix $\mathbf{P}$ is not only to help lower out-of-band emissions, but also to eliminate components that would otherwise appear in the upper right and lower left corners of $\mathbf{C}_{n, a}$ [10].

\section{Low COST EQUALISATION}

\section{A. MMSE and ZF Equalisation}

Assuming perfect knowledge of the channel matrix $\mathbf{H}_{n}$, the approach in [12] can be extended to the system in Fig. 1. In the ideal case, a linear block MMSE equaliser is defined based on the system matrix $\mathbf{C}_{n, a}$. Below, we restrict the calculation of $\mathbf{W}_{n}$ to the first $Q$ sub- and super-diagonals of $\mathbf{C}_{n, a}$ by means of a binary masking matrix $\mathbf{M}$ with elements

$$
[\mathbf{M}]_{i j}= \begin{cases}1 & 0 \leq|i-j| \leq Q, \\ 0 & Q<|i-j|<N_{\mathrm{a}} .\end{cases}
$$

The shape of this matrix as shown in Fig. 2 is imprinted on the masked matrix $\mathbf{B}_{n}=\mathbf{M} \odot \mathbf{C}_{n}$, where $\odot$ represents elementwise multiplication. Based on the masked matrix, analogously to [12] the MMSE equaliser can be defined as

$$
\mathbf{W}_{n, \mathrm{MMSE}}=\mathbf{B}_{n}^{\mathrm{H}}\left(\mathbf{B}_{n} \mathbf{B}_{n}^{\mathrm{H}}+\gamma^{-1} \mathbf{I}\right)^{-1},
$$

where $\gamma$ is the signal to noise ratio (SNR) at the input to the equaliser, assuming corruption by white Gaussian noise. The matrix inversion in (10) requires $\mathcal{O}\left(N_{a}^{3}\right)$ flops which is not practical for high values of $N_{a}$, such as found in digital video broadcast standards [21]. 


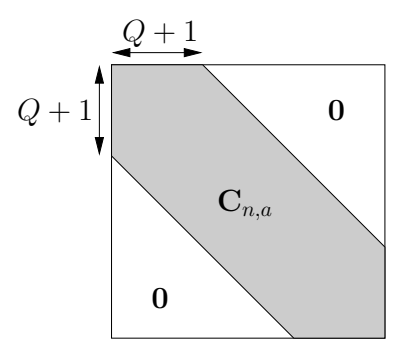

Figure 2. Structure of the modified channel matrix $\mathbf{C}_{n, a}$, where only the shaded areas, consisting of the first $Q$ off-diagonals, are retained for processing.

The zero-forcing (ZF) equaliser $\mathbf{W}_{n, \mathrm{ZF}}$ can be calculated from (10) for the special case

$$
\mathbf{W}_{n, \mathrm{ZF}}=\left.\mathbf{W}_{n, \mathrm{MMSE}}\right|_{\gamma \rightarrow \infty} .
$$

Similar to (10), the matrix inversion implicit in (11) is of order $\mathcal{O}\left(N_{a}^{3}\right)$.

\section{B. Low Cost Approaches}

The masking of the channel matrix $\mathbf{C}_{n, a}$ by $\mathbf{M}$ in (9) is justified since the equivalent channel matrix in the fractional domain is approximately banded [6]. With $\mathbf{B}_{n}$ restricted to $Q$ off-diagonal terms, the product $\mathbf{B}_{n} \mathbf{B}_{n}^{\mathrm{H}}$ is also banded with a structure similar to that in Fig. 2, but with a maximum of $2 Q$ off-diagonal terms above and below the main diagonal.

This band structure has previously been exploited for similar OFDM equalisation schemes using $\mathbf{L D L}^{\mathrm{H}}$ [17] or LSQR [14] factorisations of the Hermitian term $\mathbf{G}_{n}=\mathbf{B}_{n} \mathbf{B}_{n}^{\mathrm{H}}+\gamma^{-1} \mathbf{I}$. Here, we utilise the sparsity of $\mathbf{G}_{n}$ to invoke a very recently reported iterative least squares minres (LSMR) approach in [18], which claims to offer lower complexity, higher numerical stability, and faster convergence than the LSQR method.

\section{Complexity Consideration}

Although the MMSE outperforms other linear equalisers [11], the matrix inversion in (10) requires a substantial number of $O\left(N_{A}^{3}\right)$ flops [17], which is forbidding for large $N_{a}$. By appling an $\mathbf{L D L}{ }^{\mathrm{H}}$ factorisation to $\mathbf{G}_{n}$ in calculating either MMSE of ZF solutions in (10) and (11), the number of complex operations compared to standard matrix inversion methods such as Gaussian elimination [16] can be reduced to $\left(8 Q^{2}+22 Q+4\right) N_{A}$ complex operations [17].

The LSMR implementation of either MMSE or ZF solution requires $O\left(N_{A}(Q+1)\right)$ flops at each iteration, leading to a total of $O\left(N_{A}(Q+1) I\right)$ flops if the number of iteration steps is limited to $I$ [18]. The cost and storage requirement per iteration step is listed in Tab. I, allowing a comparison to the iterative $\mathrm{LSQR}$ approach. From the table, it is clear that LSQR has similar storage requirements and complexity than LSMR, but the latter can achieve the same accuracy of inversion with a considerably lower number of iterations, hence leading to an overall saving in complexity. From Tab. I it is clear that both LSQR and LSMR have a considerably lower complexity than e.g. direct matrix inversion or even matrix inversion via an $\mathbf{L D L}^{\mathrm{H}}$ factorisation.
Table I

COMPUTATION AND STORAGE REQUIREMENTS FOR LSQR AND LSMR ALGORITHMS

\begin{tabular}{|c|c|c|c|}
\hline & Vector Storage & Matrix Storage & Computations per iteration \\
\hline \hline LSMR & $4 \times N_{A}$ & $2\left(N_{A} \times N_{A}\right)$ & $\approx O\left(N_{A}(Q+1)\right)$ \\
\hline LSQR & $3 \times N_{A}$ & $2\left(N_{A} \times N_{A}\right)$ & $O\left(N_{A}(Q+1)\right)$ \\
\hline
\end{tabular}

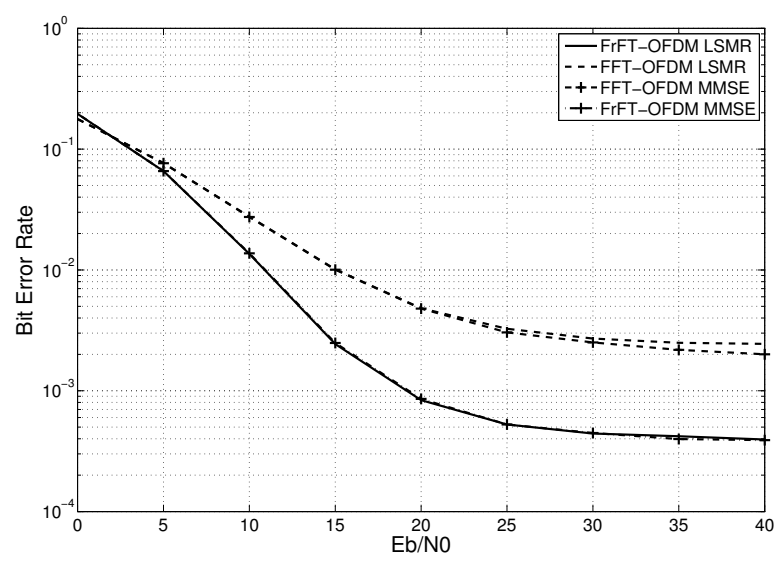

Figure 3. Uncoded BER performance comparison between FrFT-OFDM and FFT-OFDM with $Q=5$, using both standard inversion and the LSMR algorithm to implement an MMSE design.

In the next section, we will compare the different computational methods, whereby MMSE and ZF solutions, unless explicitly connected with either LSQR or LSMR, are assumed to be computed by an $\mathbf{L D L}^{\mathrm{H}}$ factorisation.

\section{Simulations And Results}

The uncoded BER performance of the proposed FrFTOFDM with LSMR equalisation is investigated below by means of simulation. We assume an FrFT-OFDM system with $N=128$ subcarriers, $N_{\mathrm{a}}=96$ active subcarriers, cyclic prefix length $L=8$, and QPSK modulation. The transmission is simulated over Rayleigh fading channels with an exponential power delay profile and root-mean-square delay spread of 3 sampling periods. The temporal variation of the channel is governed by a Doppler spread $\Omega_{\mathrm{D}}=0.15 \Delta \Omega$, where $\Delta \Omega$ is the normalised subcarrier spacing. Simulations are performed over $10^{4}$ randomly drawn channels, each simulated for one block period.

In Fig.3, we compare the FrFT-OFDM with the classic OFDM using an equaliser restricted to operating on the first $Q=5$ off-diagonal elements of a perfectly known channel matrix. The performance of all systems levels out for high $\mathrm{Eb} / \mathrm{No}$ due to the error in omitting off-diagonals greater than $Q=5$. Since in the FrFT case most of the energy within the channel matrix is concentrated around the main diagonal, it can achieve a better performance than FFT-based OFDM systems despite a very similar complexity. The approached labelled MMSE represent a standard inversion of the approximate channel matrix $\mathbf{C}_{n, a}$, while the LSMR approach implements an MMSE design but with the reduced complexity of the iterative LSMR algorithm [18]. 


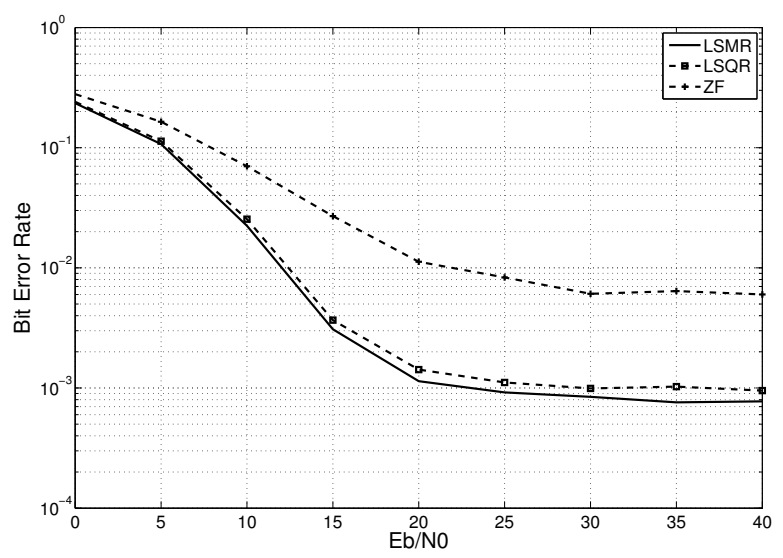

Figure 4. Uncoded BER Comparison for FrFT-OFDM based on a ZF inversion of $\mathbf{C}_{n, a}$ with $Q=5$ using standard inversion (ZF), LSQR, and LSMR approaches.

Concentrating on the FrFT-OFDM system, we demonstrate different approaches of the ZF design, including a standard inversion method, as well as LSQR and LSMR approaches to iteratively solve the inversion. Performance characteristics for the three systems are shown in Fig. 4. The standard matrix inversion for the ZF design is prone to numerical instabilities if the matrix $\mathbf{C}_{n, a}$ is ill-conditioned. This results in a relatively poor ZF design using the plain matrix inversion approach. Both LSQR and LSMR exhibit significantly improved stability, whereby the LSMR method achieves a small advantage due to its compatibility with sparse and ill-conditioned systems.

Three different MMSE equalisers for the proposed FrFTOFDM system are assessed in Fig. 5. Due to regularisation, the instability in the case of direct inversion is less problematic than for the ZF case shown in Fig. 4, and all three approaches - direct inversion, as well as LSQR and LSMR solutions to the regularised problem - perform almost identically. The difference lies in the complexity, whereby both LSQR and LSMR are reduced complexity methods, with LSMR requiring a lower number of iterations to reach the same accuracy of the LSQR approach.

\section{CONCLUSIONS}

We have considered an FrFT-based multicarrier system akin to OFDM, whereby the FrFT replacement of the FFT has distinct advantages when operating in doubly-dispersive channels. There, the FrFT has coefficients that remain largely localised around the main diagonal, while for DFT-OFDM the diagonalisation quickly degrades in Doppler scenarios due to energy leakage across the channel matrix.

Against this background, we have compared a number of equalisation strategies, whereby iterative techniques based on the LSQR or LSMR algorithms offer significant advantages over standard inversion. Specifically, for ZF designs both LSQR and LSMR offer a considerably enhanced performance due to numerical robustness, with a slight advantage to LSMR. In MMSE designs, all equalisation approaches perform linearly, but the proposed approach offers particularly low com-

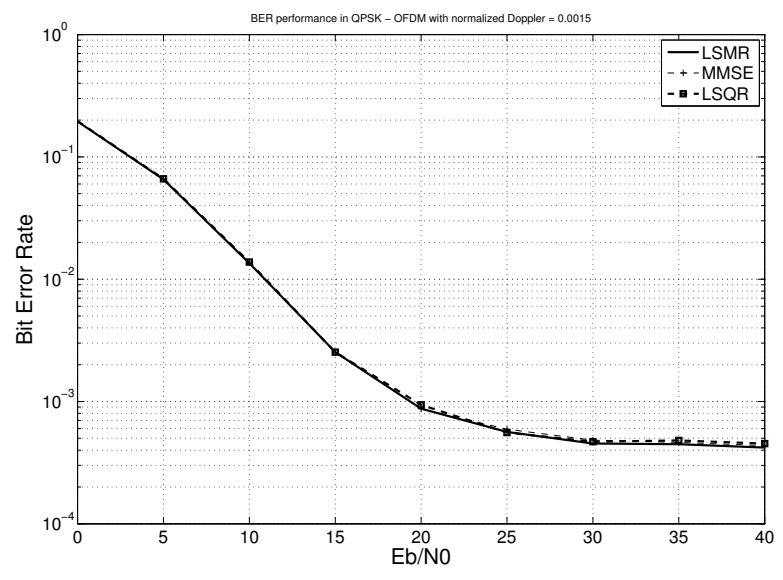

Figure 5. Uncoded BER Comparison for FrFT-OFDM based on an MMSE equalisation of $\mathbf{C}_{n, a}$ with $Q=5$ using standard regularised inversion (MMSE), LSQR, and LSMR approaches.

plexity because of its faster convergence and lower number of iterations required.

\section{REFERENCES}

[1] P. Robertson and S. Kaiser, "Analysis of the loss of orthogonality through doppler spread in OFDM systems," in Global Telecommunications Conference, vol. 1B, Rio de Janeiro, Brazil, 1999, pp. 701-706.

[2] T. Wang, J. Proakis, E. Masry, and J. Zeidler, "Performance degradation of OFDM systems due to Doppler spreading," IEEE Transactions on Wireless Communciations, vol. 5, no. 6, pp. 1422-1432, June 2006.

[3] I. Barhumi, G. Leus, and M. Moonen, "Equalization for OFDM over doubly selective channels," IEEE Transactions on Signal Processing, vol. 54, no. 4, pp. 1445 1458, april 2006.

[4] A. Scaglione, G. B. Giannakis, and S. Barbarossa, "Redundant Filterbank Precoders and Equalizers. I. Unification and Optimal Designs," IEEE Transactions on Signal Processing, vol. 47, no. 7, pp. 1988-2006, July 1999.

[5] — " "Filterbank Transceivers Optimizing Information Rate in Block Transmission over Dispersive Channels," IEEE Transactions on Information Theory, vol. 45, no. 4, pp. 1019-1032, April 1999.

[6] M. Martone, "A multicarrier system based on the fractional Fourier transform for time-frequency-selective channels," IEEE Transactions on Communications, vol. 49, no. 6, pp. 1011-1020, June 2001.

[7] E. Chen, R. Tao, and X. Meng, "The OFDM system based on the fractional Fourier transform," in First International Conference on Innovative Computing, Information and Control, vol. 3, September 2006, pp. 14-17.

[8] D. Stojanovic, I. Djurovic, and B. Vojcic, "Interference analysis of multicarrier systems based on affine Fourier transform," IEEE Transactions on Wireless Communications, vol. 8, no. 6, pp. 2877-2880, June 2009.

[9] J. Zheng and Z. Wang, "ICI analysis for FrFT-OFDM systems to frequency offset in time-frequency selective 
fading channels," IEEE Communications Letters, vol. 14, no. 10, pp. 888-890, October 2010.

[10] P. Schniter, "Low-complexity equalization of OFDM in doubly selective channels," IEEE Transactions on Signal Processing, vol. 52, no. 4, pp. 1002-1011, April 2004.

[11] Y.-S. Choi, P. Voltz, and F. Cassara, "On channel estimation and detection for multicarrier signals in fast and selective Rayleigh fading channels," IEEE Transactions on Communications, vol. 49, no. 8, pp. 1375-1387, August 2001.

[12] L. Rugini, P. Banelli, and G. Leus, "Simple equalization of time-varying channels for OFDM," IEEE Communications Letters, vol. 9, no. 7, pp. 619-621, July 2005.

[13] T. Hrycak and G. Matz, "Low-complexity time-domain ICI equalization for OFDM communications over rapidly varying channels," in Fortieth Asilomar Conference on Signals, Systems and Computers, Pacific Grove, CA, November 2006, pp. 1767-1771.

[14] G. Tauböck, M. Hampejs, P. Svac, G. Matz, F. Hlawatsch, and K. Gröchenig, "Low-complexity ICI/ISI equalization in doubly dispersive multicarrier systems using a decision-feedback LSQR algorithm," IEEE Transactions on Signal Processing, vol. 55, no. 5, pp. 2432-2436, May 2011.

[15] C. C. Paige and M. A. Saunders, "LSQR: An algorithm for sparse linear equations and sparse least squares," ACM Transactions on Mathematical Software, vol. 8, no. 1, pp. 43-71, March 1982.

[16] G. H. Golub and C. F. Van Loan, Matrix Computations, 3rd ed. Baltimore, Maryland: John Hopkins University Press, 1996.

[17] L. Rugini, P. Banelli, and G. Leus, "Low-complexity banded equalizers for OFDM systems in Doppler spread channels," EURASIP Journal on Applied Signal Processing, vol. 2006, pp. 1-13, 2006.

[18] D. C.-L. Fong and M. A. Saunders, "LSMR: An iterative algorithm for sparse least-squares problems," SIAM Journal on Scientific Computing, March 2011, (submitted).

[19] C. Candan, M. Kutay, and H. Ozaktas, "The discrete fractional Fourier transform," IEEE Transactions on Signal Processing, vol. 48, no. 5, pp. 1329-1337, May 2000.

[20] E. Chen, P. Ma, L. Qi, and R. Tao, "Multi-tap equalization algorithm for the OFDM system based on the fractional Fourier transform," in Fourth International Conference on Communications and Networking in China, August 2009, pp. 1-4.

[21] U. Ladebusch and C. Liss, "Terrestrial DVB (DVB-T): A broadcast technology for stationary, portable, and mobile use" Proceedings of the IEEE, vol. 94, no. 1, pp. 183193, January 2006. 